\title{
Cadmium Intake, Dietary Patterns and Hyperuricemia Among Adults in China
}

\author{
Zumin Shi ${ }^{1}$ [D
}

Received: 6 February 2020 / Revised: 2 June 2020 / Accepted: 4 August 2020 / Published online: 17 August 2020

(c) The Author(s) 2020

\begin{abstract}
Blood and urine cadmium levels have been found to be positively associated with hyperuricemia (HU) in some studies. Few studies have examined the association between dietary cadmium intake, dietary patterns and HU in the Chinese population. The study analysed data from 8429 adults who participated in the China Health and Nutrition Survey (CHNS). Dietary intake was assessed by 3-day $24 \mathrm{~h}$ food record in seven waves of survey (1991, 1993, 1997, 2000, 2004, 2006 and 2009). Serum uric acid was measured in 2009. Dietary pattern was identified using factor analysis. The association between cumulative dietary cadmium intake, dietary patterns and HU was assessed using logistic regression. In total, 1737 (16.3\%) participants had $\mathrm{HU}$ in the study sample. After adjusting for demographic, lifestyle factors (i.e. smoking, alcohol drinking, physical activity) and chronic conditions, the OR for HU was 2.25 (95\% CI 1.84-2.77, $p$ for trend <0.001) for extreme quartiles of estimated cumulative cadmium intake. A traditional southern dietary pattern characterized by high intake of rice, pork, fish and vegetables, and low intake of wheat was associated with three times increased prevalence of HU; comparing the extreme quartiles, the odds ratio (OR) was 3.24 (95\% CI 2.61-4.01). No association between the modern dietary pattern (high intake of fruit, soymilk, egg, milk and deep fried products) and HU was found. In conclusion, traditional southern dietary pattern and cadmium intake are positively associated with hyperuricemia among Chinese adults.
\end{abstract}

Keywords Cadmium intake $\cdot$ Dietary pattern $\cdot$ Hyperuricemia $\cdot$ Chinese

\section{Introduction}

Hyperuricemia (HU) is a common metabolic disease that affects a high proportion of the population in both developed and developing counties. The prevalence of $\mathrm{HU}$ was $21 \%$ in both men and women in the USA (Zhu et al. 2011). In China, $21.6 \%$ of men and $8.6 \%$ of women had HU (Liu et al. 2011). There is an increasing body of evidence that suggests HU increases the risk of gout and other chronic non-communicable diseases (NCDs) including diabetes, cardiovascular disease and cancer (Wu et al. 2017).

Electronic supplementary material The online version of this article (https://doi.org/10.1007/s12403-020-00375-4) contains supplementary material, which is available to authorized users.

Zumin Shi

zumin@qu.edu.qa

1 Human Nutrition Department, College of Health Sciences, QU Health, Qatar University, P.O. Box 2713, Doha, Qatar
For centuries, diet has been identified as a risk factor for gout (Nuki and Simkin 2006). Diet rich in purine (such as meats, seafood, purine-rich vegetables and animal protein) has been shown to be associated with gout in some studies (Choi et al. 2004). Soft drink and alcohol consumption has also been found to be positively associated with HU (Choi and Curhan 2004; Choi et al. 2008). Adherence to Mediterranean diet or Dietary Approaches to Stop Hypertension (DASH) diet is associated with a lower likelihood of HU (Juraschek et al. 2016; Kontogianni et al. 2012). Several regional studies suggest that overall dietary patterns are associated with HU in China (Liu et al. 2018; Xia et al. 2018; Zhang et al. 2012). For example, 'animal products' dietary pattern was positively associated with $\mathrm{HU}$ in the Yi ethnic group (Liu et al. 2018). A 'meat food pattern' was positively but a 'traditional Chinese pattern' was inversely associated with $\mathrm{HU}$ in a cross-sectional study conducted in Zhejiang Province, China (Zhang et al. 2012).

Cadmium is nephrotoxic and increases the risk of chronic kidney diseases (Ferraro et al. 2010). Food intake is the most significant source of cadmium exposure in the 
general population. An excessive cadmium exposure from the diet in China has caused concern (He et al. 2013; Yuan et al. 2014). Recent studies suggest that cadmium is associated with diabetes and chronic kidney disease (CKD) in the Chinese population (Shi et al. 2018a). A limited number of studies have examined the association between blood/urine cadmium and HU with inconsistent findings (Arrebola et al. 2019; Jung et al. 2019; Krishnan et al. 2012; Sun et al. 2017). Data from the National Health and Nutrition Examination Survey (NHANES) suggested a null association between blood cadmium and HU (Krishnan et al. 2012). In Spain, urine cadmium was found to be positively associated with HU (Arrebola et al. 2019). A regional study from China found that blood cadmium was associated with an increased likelihood of having HU in men but not in women (Sun et al. 2017). However, the study did not have information on cadmium intake. It is unknown whether cadmium from foods increases the risk of HU. In a previous study, it has been shown that both traditional rice-based dietary pattern and cadmium intake were associated with CKD among participants who attended the Chinese Health and Nutrition Survey (CHNS) (Shi et al. 2018a).

Using data from the CHNS, this study aimed to assess the association between dietary patterns, intake of cadmium from foods and HU among Chinese adults.

\section{Method}

\section{Study Design and Sample}

CHNS is an open cohort started in 1989 and uses a multistage random-cluster sampling process to select households in both urban and rural areas that includes nine provinces in China (Zhai et al. 2014). All the members in the selected household were invited to participate in the study, as well as the follow-up surveys in every $2-4$ years. Blood samples were collected only in the 2009 survey. The survey was approved by the institutional review committees of the University of North Carolina and the National Institute of Nutrition and Food Safety. All participants gave informed consent. In the current analysis, dietary and lifestyle information between 1991 and 2009 were used.

In total, 9551 of the 18,887 participants in the 2009 survey had blood measurements for serum uric acid and renal function. For the purpose of this study, participants who were under 18 years of age, or without dietary information in 2009, pregnant women and those with extreme energy intake (men: $>6000 \mathrm{kcal}$ or $<800 \mathrm{kcal}$; women: $>4000$ or $<600 \mathrm{kcal}$ ), were excluded. The final analytical sample included 8429 adults.

\section{Measurements}

\section{Outcome Measures: Serum Uric Acid and HU}

Serum uric acid was measured by an enzymatic colorimetric method with a Hitachi 7600 automated analyser (Hitachi Inc., Tokyo, Japan) using determiner reagents (Randox Laboratories Ltd., Crumlin, UK). HU was defined as serum uric acid concentrations $\geq 7 \mathrm{mg} / \mathrm{dL}$ in men and $\geq 6 \mathrm{mg} / \mathrm{dL}$ in women ( $\mathrm{Li}$ et al. 2018).

\section{Exposure Measures: Dietary Patterns and Cadmium Intake}

The study used a comprehensive 3-day $24 \mathrm{~h}$ food recall method to measure the dietary intake. The detailed description of the dietary measurement has been published elsewhere (Zhai et al. 2014). At each wave, trained interviewers weighed all foods and condiments in the home inventory, purchased from markets or picked from gardens, and food waste during the 3-day survey period. The individual dietary intake on each of the 3 consecutive days was interviewed and supported by dietary records kept by the individual. Food consumption data were converted into nutrient intake using the Chinese Food Composition Table.

In the dietary pattern analysis, food intakes were first collapsed into 35 food groups based on similar nutrient profiles or culinary use, and average food intake for individuals (gram/day) calculated for each wave. Dietary patterns across the seven waves (1991-2009) were identified by factor analysis, using the standard principal component analysis method. The number of factors retained was determined by the following criteria: (1) eigenvalue $>1$; (2) scree plot; and (3) factor interpretability. Factors were rotated with an orthogonal (varimax) rotation to improve interpretability and minimize the correlation between the factors. Labelling of the factors was subjective and based on the interpretation of the pattern structures. At each wave, participants were assigned pattern-specific factor scores based on the factor loadings of each food group.

In estimating cadmium intake, this study used food cadmium composition table from published literature in China (Yuan et al. 2014). The food cadmium composition (mg/ $\mathrm{kg}$ ) table was based on the measured cadmium levels in 2629 food samples randomly purchased from the market in 31 provinces in 2012 in China. The limit of detection (LOD) and the limit of quantification (LOQ) for cadmium were $0.1 \mu \mathrm{g} \mathrm{kg}^{-1}$ and $0.3 \mu \mathrm{g} \mathrm{kg}^{-1}$, respectively.

The cumulative mean dietary intake score between 1991 and 2009 was used as it better reflects a long-term diet and may reduce dietary measurement error. This approach has been used in other studies (Lin et al. 2011). Cumulative 
mean cadmium intake, as well as cumulative mean intake of individual foods, was recoded into quartiles.

\section{Covariates}

In the analysis sociodemographic, lifestyle factors and chronic conditions in 2009 were treated as covariates. Physical activity level (metabolic equivalent of task, (MET)) was calculated based on self-reported four major activities (including occupational, domestic, transportation and leisure time physical activity) and duration using a Compendium of Physical Activities. Self-reported smoking status was recoded as non-smokers, ex-smokers and current smokers. A constructed urbanization index was used to reflect residence and recoded into tertiles (Zhai et al. 2014). Education and per capita annual family income were recoded into three levels (low, medium and high). Hypertension was defined as systolic blood pressure above $140 \mathrm{mmHg}$ and/or diastolic blood pressure above $90 \mathrm{mmHg}$, or having known hypertension. Overweight was defined as BMI $\geq 24 \mathrm{~kg} / \mathrm{m}^{2}$.

Participants' blood samples were tested for fasting plasma glucose (FPG) and glycated haemoglobin (HbA1c) (Yan et al. 2012). C-reactive protein (CRP) was measured in blood using the immunoturbidimetric method with Denka Seiken, Japan reagents. Diabetes was defined as FPG $>7.0 \mathrm{mmol} / \mathrm{l}$, $\mathrm{HbA} 1 \mathrm{C}>6.5$ or having known diabetes (self-reported doctor diagnosed). Kidney function was evaluated by the estimated glomerular filtration rate (eGFR). Serum creatinine was measured using Jaffe's kinetic method (Hitachi 7600 automated analyser, Hitachi Inc., Tokyo, Japan). CKDEPI creatinine equation was used to estimate eGFR (Inker et al. 2012). Chronic kidney disease (CKD) was defined as $\mathrm{eGFR}<60 \mathrm{~mL} / \mathrm{min} / 1.73 \mathrm{~m}^{2}$.

\section{Statistical Analyses}

The Chi-square test was used to compare differences between groups for categorical variables and ANOVA for continuous variables. Multivariable logistic regression was used to assess the association between dietary pattern and HU. The first quartile (lowest intake) of each dietary exposure variable was used as the reference group. Four stepwise multivariable models adjusted for either known risk factors for HU or sociodemographic factors were used: Model 1 adjusted for age, gender and energy intake (continuous); Model 2 further adjusted for education, income (low, medium and high), urbanization level (low, medium and high), physical activity, smoking, alcohol drinking; Model 3 further adjusted for overweight/obesity, hypertension and diabetes; Model 4 further adjusted for CKD. In a sensitivity analysis, only those subjects with seven waves of dietary measurements were included $(n=3298)$. To assess whether cadmium intake mediates the association between dietary patterns and $\mathrm{HU}$, dietary cadmium intake was included in the full model (model 3). Four similar models were used to assess the association between cumulative cadmium intake and HU. In order to better understand the relationship between the overall dietary pattern, cadmium and HU, the associations between intake of individual foods and $\mathrm{HU}$ were assessed using logistic regression models with adjustment for age, gender, energy intake, education, income, urbanization level, physical activity, smoking and alcohol drinking.

In the analysis, multiplicative interaction between sociodemographic factors and dietary patterns/cadmium intake was tested by including a cross-product term in the main multivariable model (model 3). All the analyses were performed by using STATA 16.1 (Stata Corporation, College Station). Statistical significance was considered when $p<0.05$ (two sided).

\section{Results}

\section{Overall Sample Characteristics}

The mean age was 51.0 (SD 15.0) years among 8429 participants (3982 men and 4447 women) in 2009. The median number of dietary measurements was five (interquartile range 2-7). The prevalence of $\mathrm{HU}$ was $16.3 \%$ (22.2\% in men and $11.9 \%$ in women). Overweight/obesity, hypertension, diabetes and CKD were positively associated with HU (Supplement Fig. 1). Income was not associated with HU. The cumulative mean intake of dietary cadmium was $26.6 \mu \mathrm{g} / \mathrm{d}$ (SD 9.8). Across the quartiles of the cadmium intake, there was a significant increase of $\mathrm{HU}, \mathrm{CKD}$, intake of tradition dietary pattern and macronutrients (Table 1).

\section{Dietary Patterns Constructed}

Two dietary patterns were constructed using factor analysis and explained $11.7 \%$ of the variance in food intake. The "traditional southern" dietary pattern had high factor loadings of rice, pork and vegetable, but with inverse loadings for wheat flour and whole grain (Table 2). The modern dietary pattern had a high intake of fruit, soy milk, eggs, milk, deep fried products, fast food and cakes, and inverse loadings for rice and salted vegetables. Supplement Fig. 2 shows the food intake by quartiles of dietary patterns. Across quartiles of the "traditional southern" dietary pattern, there was a significant increase in the intake of rice, pork and fish. Average wheat intake was lower among those with a high intake of the traditional southern pattern. For the "modern" dietary pattern, the mean fruit intake increased with quartile, while the mean rice intake monotonically decreased with quartile. The traditional south dietary pattern was positively but 
Table 1 Sample characteristics in 2009 by quartiles of cumulative cadmium intake among adutls attending the China Health and Nutrition Survey $(\mathrm{N}=8429)$

\begin{tabular}{|c|c|c|c|c|c|}
\hline & $\begin{array}{l}\mathrm{Q} 1 \\
N=2108\end{array}$ & $\begin{array}{l}\mathrm{Q} 2 \\
N=2107\end{array}$ & $\begin{array}{l}\text { Q3 } \\
N=2107\end{array}$ & $\begin{array}{l}\mathrm{Q} 4 \\
N=2107\end{array}$ & $p$-value \\
\hline Traditional dietary pattern, mean (SD) & $-0.8(0.6)$ & $-0.1(0.7)$ & $0.4(0.6)$ & $0.8(0.7)$ & $<0.001$ \\
\hline Modern dietary pattern, mean (SD) & $0.6(1.2)$ & $0.6(1.3)$ & $0.4(1.1)$ & $0.4(1.1)$ & $<0.001$ \\
\hline Energy intake (kcal/d), mean (SD) & $1915.2(589.7)$ & $2047.3(600.9)$ & $2179.3(605.3)$ & $2385.8(658.1)$ & $<0.001$ \\
\hline Fat intake (g/d), mean (SD) & $59.8(30.4)$ & $71.6(32.4)$ & $76.7(34.2)$ & $89.0(39.0)$ & $<0.001$ \\
\hline Protein intake (g/d), mean (SD) & $58.1(20.2)$ & $63.1(21.9)$ & $67.8(22.2)$ & $74.9(23.7)$ & $<0.001$ \\
\hline Carbohydrate intake (g/d), mean (SD) & $283.4(101.7)$ & $282.8(98.1)$ & $298.7(98.1)$ & $314.5(105.8)$ & $<0.001$ \\
\hline Intake of cadmium $(\mu \mathrm{g} / \mathrm{d})$, mean $(\mathrm{SD})$ & $14.6(3.0)$ & $22.9(2.2)$ & $29.7(1.9)$ & $39.2(6.1)$ & $<0.001$ \\
\hline Age (years), mean (SD) & $52.0(16.2)$ & $51.6(15.8)$ & $51.0(14.7)$ & $49.4(13.0)$ & $<0.001$ \\
\hline BMI $\left(\mathrm{kg} / \mathrm{m}^{2}\right)$, mean $(\mathrm{SD})$ & $23.8(3.5)$ & $23.4(3.6)$ & $23.1(3.5)$ & $23.2(3.3)$ & $<0.001$ \\
\hline $\operatorname{Men}(\%)$ & $772(36.6 \%)$ & $881(41.8 \%)$ & $960(45.6 \%)$ & $1,369(65.0 \%)$ & $<0.001$ \\
\hline Low income (\%) & $750(35.9 \%)$ & $549(26.4 \%)$ & $566(27.1 \%)$ & $499(24.0 \%)$ & $<0.001$ \\
\hline Low education $(\%)$ & $1008(47.9 \%)$ & $872(41.4 \%)$ & $903(43.0 \%)$ & $717(34.1 \%)$ & $<0.001$ \\
\hline Hypertension (\%) & $632(30.1 \%)$ & $626(30.0 \%)$ & $535(25.7 \%)$ & $501(24.1 \%)$ & $<0.001$ \\
\hline Diabetes (\%) & $279(13.2 \%)$ & $250(11.9 \%)$ & $181(8.6 \%)$ & $194(9.2 \%)$ & $<0.001$ \\
\hline Urbanization (\%) & & & & & $<0.001$ \\
\hline Low & $1006(47.7 \%)$ & $640(30.4 \%)$ & $587(27.9 \%)$ & $607(28.8 \%)$ & \\
\hline Medium & $591(28.0 \%)$ & $767(36.4 \%)$ & $746(35.4 \%)$ & $689(32.7 \%)$ & \\
\hline High & $511(24.2 \%)$ & $700(33.2 \%)$ & $774(36.7 \%)$ & $811(38.5 \%)$ & \\
\hline Smoking (\%) & & & & & $<0.001$ \\
\hline Non smoker & $1545(73.3 \%)$ & $1509(71.7 \%)$ & $1505(71.5 \%)$ & $1236(58.7 \%)$ & \\
\hline Ex-smokers & $72(3.4 \%)$ & $67(3.2 \%)$ & $76(3.6 \%)$ & $67(3.2 \%)$ & \\
\hline Current smokers & $491(23.3 \%)$ & $529(25.1 \%)$ & $524(24.9 \%)$ & $803(38.1 \%)$ & \\
\hline High sensitivity CRP (mg/dl), median (IQR) & $1.0(0.0-2.0)$ & $1.0(0.0-2.0)$ & $1.0(0.0-2.0)$ & $1.0(1.0-2.0)$ & 0.14 \\
\hline CKD $(\%)$ & $110(5.2 \%)$ & $165(7.8 \%)$ & $186(8.8 \%)$ & $180(8.5 \%)$ & $<0.001$ \\
\hline Physical activity (MET hours/week), mean (SD) & $125.7(116.4)$ & $126.7(111.8)$ & $116.6(100.6)$ & $125.4(100.4)$ & 0.010 \\
\hline Hyperuricemia $(\%)$ & $227(10.8 \%)$ & $319(15.1 \%)$ & $376(17.9 \%)$ & $451(21.4 \%)$ & $<0.001$ \\
\hline
\end{tabular}

the modern pattern was inversely associated with cadmium intake (Supplement Fig. 3).

\section{Association Between Cadmium Intake and HU}

Cadmium intake was positively associated with serum uric acid after adjusting for age and gender (Supplement Fig. 4). In multivariable model adjusting for sociodemographic and lifestyle factors as well as overweight/obesity, hypertension and diabetes, the odds ratio (OR) $(95 \% \mathrm{CI})$ for $\mathrm{HU}$ across quartiles of cadmium intake was 1.00 (reference), 1.48, 1.94 and 2.25(1.84-2.77) ( $p$ for trend <0.001) (Table 3). The association remained after further adjusting for CKD but the association became stronger when the analysis was limited to those who attended all seven waves of dietary survey $(n=3298)$. Excluding those with CKD or adjusting for CRP the association between cadmium and HU remained (data not shown). The above association remained unchanged when the modern dietary pattern was further adjusted (data not shown). However, the association between cadmium intake and HU disappeared when both traditional southern dietary pattern and modern pattern were adjusted with ORs across quartiles of cadmium intake being 1.00, 1.01, 0.98 and $0.87(0.64-1.18)$ ( $p$ for trend 0.273).

\section{Association Between Dietary Patterns and HU}

The prevalence of HU in 2009 increased across quartiles of the cumulative traditional southern dietary pattern were $9.4 \%, 15.8 \%, 18.1 \%$ and $21.9 \%$. After adjusting for age, gender, income, education, urbanization, intake of energy, smoking, alcohol drinking, physical activity, diabetes, hypertension and overweight/obesity (model 3), across quartiles of the traditional southern dietary pattern ORs for HU were 1.00, 2.09, 2.65, and 3.24 (95\% CI 2.61-4.01) ( $p$ for trend $<0.001$ ), respectively. Further adjustment for CKD attenuated the association slightly. The association became stronger when the analysis was limited to those who attended all seven waves of dietary measurements: ORs were $1.00,265,2.90$ and 4.14 (95\% CI 2.87-5.97) across 
Table 2 Factor loadings of identified dietary patterns

\begin{tabular}{lll}
\hline & Traditional pattern & Modern pattern \\
\hline Rice & 0.78 & -0.21 \\
Wheat & -0.72 & - \\
Whole grain & -0.44 & - \\
Pork & 0.41 & 0.31 \\
Fish & 0.35 & 0.28 \\
Fresh vegetable & 0.29 & - \\
Dry tofu & 0.27 & - \\
Offal & 0.22 & - \\
Fruit & - & 0.46 \\
Milk & - & 0.41 \\
Eggs & - & 0.39 \\
Fast food & - & 0.39 \\
Soy milk & - & 0.38 \\
Deep fried products & -0.29 & 0.35 \\
Fungus & - & 0.31 \\
Cake & - & 0.30 \\
Poultry & 0.24 & 0.27 \\
Shrimp & - & 0.26 \\
Nuts & - & 0.24 \\
Beer & - & 0.23 \\
Beef & - & 0.21 \\
Variance explained $(\%)$ & 6.2 & 5.5 \\
\hline
\end{tabular}

Bean thread noodle, Tofu, salted vegetables, lamb, tubers, yoghurt, beverage, legume, spirit, sugar, milk powder, fresh bean, wine and others had factors loadings $<|0.2|$

quartiles of this pattern. However, adjusting for cadmium intake decreased the ORs slightly (for quartile four, the OR decreased from 3.24 to 3.03 , corresponding to a $6.5 \%$ decrease in effect estimate).

No association between the modern dietary pattern and $\mathrm{HU}$ was found in the fully adjusted model (model 3 ). Comparing the extreme quartiles of the cumulative modern dietary pattern, the OR for CKD were 1.14 (95\%CI 0.90-1.43) for quartile 4 compared to quartile 1 . The above associations were not changed after further adjusting for CRP (data not shown).

\section{Association Between Individual Foods Intake and $\mathrm{Hu}$}

Table 4 shows the association between cumulative mean individual food intake and HU. After adjusting for sociodemographic and lifestyle factors, the consumption of rice, pork, fish and poultry was positively associated with HU. However, the intake of wheat, whole grain, tubers, egg and fast food was inversely associated with HU.
Overall, no interactions were observed between cadmium intake, dietary pattern and sex, hypertension, diabetes, obesity or CKD (data not shown).

\section{Discussion}

In this population-based study of $\sim 8500$ Chinese adults, the traditional southern dietary pattern with a high intake of rice, pork, fish and vegetables, and low intake of wheat was positively associated with $\mathrm{HU}$ after adjusting for lifestyle factors, obesity, hypertension and diabetes. No association was observed between the modern dietary pattern and HU. Intake of cadmium was positively associated with the traditional southern pattern. The association between traditional dietary pattern and HU was partly mediated by CKD but not cadmium content of foods. There was a positive association between dietary cadmium and HU. However, the association between cadmium and HU was mainly due to dietary patterns.

\section{Cadmium Intake and HU}

Three studies have examined the association between blood/urine cadmium and HU so far (Arrebola et al. 2019; Sun et al. 2017). Studies in Spain and China found a positive association between blood/urine cadmium and HU (Arrebola et al. 2019; Sun et al. 2017). However, no association between blood cadmium and $\mathrm{HU}$ was found in the USA (Krishnan et al. 2012). The current study is the first to show that dietary intake of cadmium was positively associated with $\mathrm{HU}$ and the association was mainly due to the dietary patterns. The finding is consistent with another study in China which reported a positive association between blood cadmium and HU (Sun et al. 2017). In the cross-sectional SPECT-China study, comparing extreme quartiles of blood cadmium, the OR for HU was 1.61 (95\% CI 1.04-2.49) in men and 1.25 (95\% CI 0.74-2.11) in women in a model adjusted for eGFR, current smoking status, blood lead, diabetes, dyslipidaemia, hypertension and BMI. However, data from the National Health and Nutrition Examination Surveys (NHANES) study in the USA suggested no association between blood cadmium and HU (Krishnan et al. 2012). The difference may be due to the different levels of cadmium in the study populations. The blood cadmium level in adults in China is higher than in the USA (Nie et al. 2016).

The mechanism linking cadmium and HU is not fully understood. It is possible that long-term low-dose cadmium exposure may cause kidney damage through tubular dysfunction with decreased reabsorptive capacity for minerals, vitamins and other nutrients (Satarug et al. 2010). 
Table 3 Odds rations (95\% CI) for HU according to quartiles of cumulative dietary patterns and cadmium intake among Chinese adults

\begin{tabular}{|c|c|c|c|c|c|}
\hline & Q1 & Q2 & Q3 & Q4 & $p$ for trend \\
\hline \multicolumn{6}{|l|}{ Traditional southern pattern } \\
\hline Model $1^{\mathrm{a}}$ & 1 & $1.91(1.58-2.31)$ & $2.41(2.00-2.92)$ & $2.71(2.25-3.25)$ & $<0.001$ \\
\hline Model $2^{\mathrm{b}}$ & 1 & $1.88(1.53-2.32)$ & $2.15(1.74-2.67)$ & $2.59(2.11-3.18)$ & $<0.001$ \\
\hline Model $3^{\mathrm{c}}$ & 1 & $2.09(1.68-2.59)$ & $2.65(2.12-3.31)$ & $3.24(2.61-4.01)$ & $<0.001$ \\
\hline Model $3+$ CKD & 1 & $1.93(1.56-2.41)$ & $2.32(1.85-2.91)$ & $2.95(2.38-3.67)$ & $<0.001$ \\
\hline Model $3+$ dietary measures $=7$ waves & 1 & $2.95(1.90-4.58)$ & $3.40(2.32-4.99)$ & $4.75(3.31-6.81)$ & $<0.001$ \\
\hline Model $3+$ cadmium intake & 1 & $2.09(1.65-2.65)$ & $2.67(2.02-3.53)$ & $3.32(2.47-4.46)$ & $<0.001$ \\
\hline \multicolumn{6}{|l|}{ Modern dietary pattern } \\
\hline Model $1^{\mathrm{a}}$ & 1 & $1.20(1.01-1.43)$ & $1.23(1.04-1.47)$ & $1.56(1.32-1.85)$ & $<0.001$ \\
\hline Model $2^{\mathrm{b}}$ & 1 & $1.16(0.96-1.40)$ & $1.04(0.85-1.28)$ & $1.27(1.01-1.59)$ & 0.197 \\
\hline Model $3^{c}$ & 1 & $1.06(0.87-1.29)$ & $0.91(0.73-1.12)$ & $1.14(0.90-1.43)$ & 0.914 \\
\hline Model 3+CKD & 1 & $1.07(0.87-1.30)$ & $0.92(0.75-1.14)$ & $1.12(0.89-1.42)$ & 0.819 \\
\hline Model $3+$ dietary measures $=7$ waves & 1 & $0.96(0.72-1.29)$ & $0.90(0.64-1.26)$ & $1.18(0.75-1.84)$ & 0.491 \\
\hline Model $3+$ cadmium intake & 1 & $1.07(0.88-1.30)$ & $0.91(0.73-1.12)$ & $1.14(0.90-1.44)$ & 0.789 \\
\hline \multicolumn{6}{|l|}{ Cadmium intake } \\
\hline Model $1^{\mathrm{a}}$ & 1 & $1.46(1.21-1.76)$ & $1.76(1.47-2.11)$ & $2.04(1.70-2.45)$ & $<0.001$ \\
\hline Model $2^{\mathrm{b}}$ & 1 & $1.37(1.12-1.68)$ & $1.66(1.37-2.02)$ & $1.92(1.57-2.34)$ & $<0.001$ \\
\hline Model $3^{c}$ & 1 & $1.48(1.21-1.83)$ & $1.94(1.58-2.38)$ & $2.25(1.84-2.77)$ & $<0.001$ \\
\hline Model $3+$ CKD & 1 & $1.39(1.13-1.72)$ & $1.79(1.46-2.21)$ & $2.07(1.68-2.55)$ & $<0.001$ \\
\hline Model $3+$ dietary measures $=7$ waves & 1 & $1.94(1.29-2.94)$ & $3.48(2.40-5.03)$ & $3.82(2.64-5.53)$ & $<0.001$ \\
\hline Model $3+$ dietary patterns & 1 & $1.01(0.80-1.26)$ & $0.98(0.75-1.27)$ & $0.87(0.64-1.18)$ & 0.273 \\
\hline
\end{tabular}

${ }^{a}$ Model 1: adjusted for age, gender, intake of energy

${ }^{\mathrm{b}}$ Model 2: additional adjustment for education, income, urbanization level, smoking, alcohol drinking, and physical activity

${ }^{\mathrm{c}}$ Model 3: further adjustment for overweight/obesity, hypertension, and diabetes

Table 4 Odds ratio (95\%CI) for hyperuricemia by quartiles of individual food intake

\begin{tabular}{lllllr}
\hline & Q1 & Q2 & Q3 & Q4 & $p$ for trend \\
\hline Rice & 1 & $\mathbf{1 . 8 1}(\mathbf{1 . 4 8 - 2 . 2 1})$ & $\mathbf{2 . 0 1 ( 1 . 6 5 - 2 . 4 4 )}$ & $\mathbf{2 . 0 0}(\mathbf{1 . 6 4 - 2 . 4 4})$ & $<0.001$ \\
Pork & 1 & $1.01(0.82-1.23)$ & $\mathbf{1 . 3 1}(\mathbf{1 . 0 8}-\mathbf{1 . 6 0})$ & $\mathbf{1 . 5 6}(\mathbf{1 . 2 8}-\mathbf{1 . 9 0})$ & $<0.001$ \\
Vegetable & 1 & $0.95(0.79-1.14)$ & $1.08(0.90-1.29)$ & $0.89(0.74-1.07)$ & 0.455 \\
Fish & 1 & $1.00(0.81-1.22)$ & $1.15(0.96-1.36)$ & $\mathbf{1 . 4 2}(\mathbf{1 . 2 0}-\mathbf{1 . 6 9})$ & $<0.001$ \\
Poultry & 1 & $1.14(0.97-1.34)$ & $\mathbf{1 . 3 7}(\mathbf{1 . 1 8}-\mathbf{1 . 5 9})$ & - & 0.001 \\
Wheat $_{\text {Whole grain }}^{\mathrm{a}}$ & 1 & $0.87(0.74-1.03)$ & $\mathbf{0 . 7 2}(\mathbf{0 . 6 1 - 0 . 8 6})$ & $\mathbf{0 . 4 1}(\mathbf{0 . 3 4 - 0 . 5 0})$ & $<0.001$ \\
Tubers & 1 & $0.93(0.78-1.11)$ & $\mathbf{0 . 7 2}(\mathbf{0 . 6 1 - 0 . 8 4})$ & - & $<0.001$ \\
Fruit & 1 & $0.91(0.76-1.08)$ & $0.90(0.75-1.07)$ & $0.97(0.81-1.16)$ & 0.667 \\
Soy milk $^{\mathrm{b}}$ & 1 & $1.14(0.57-2.30)$ & $\mathbf{1 . 1 8}(\mathbf{1 . 0 1}-\mathbf{1 . 3 7})$ & $0.96(0.81-1.13)$ & 0.712 \\
Egg $^{\mathrm{a} g}$ & 1 & $1.06(0.92-1.23)$ & - & - & 0.420 \\
Milk $^{\mathrm{b}}$ & 1 & $0.85(0.71-1.02)$ & $0.88(0.74-1.06)$ & $\mathbf{0 . 7 9}(\mathbf{0 . 6 6 - 0 . 9 5})$ & 0.029 \\
Fast food $^{\mathrm{a}}$ & 1 & $0.88(0.73-1.06)$ & - & - & 0.185 \\
\hline
\end{tabular}

Bold values indicate the $p<0.05$

Models adjusted for age, gender, intake of energy, education, income, urbanization level, smoking, alcohol drinking, and physical activity

${ }^{a}$ Food consumption categorical levels were: none, above or below medium consumption among consumers

${ }^{\mathrm{b}}$ Food consumption categorical levels were: none consumers and consumers 
Cadmium is a well-known nephron toxic heavy metal and can cause kidney damage (Jarup et al. 2000). In a rat model, cadmium exposure increased xanthine oxidoreductase (XOR) activity and led to renal uric acid transport system dysfunction. In parallel with the urine hyperactivity of XOR, serum uric acid level increased (Wang et al. 2012). In the sample, having CKD was associated with four times increased likelihood of having HU.

Consistent with findings from the SPECT study, the association between the dietary cadmium intake and HU in the current study appears independent of chronic kidney disease. It suggests that mechanisms other than the impairment of kidney function are involved.

In the general population, food is the main source of cadmium exposure. Food can be contaminated by cadmium through modern industrial and agricultural activities (e.g. use of fertilizer and pesticides, waste disposal). In the Chinese diet, rice, vegetable, wheat and pork are the major contributors of dietary cadmium (He et al. 2013; Yuan et al. 2014). The dietary cadmium intake was much higher in China than in western countries. For example, in Sweden, the mean intake of cadmium was $19 \mu \mathrm{g} / \mathrm{d}$ in men and $13 \mu \mathrm{g} / \mathrm{d}$ in women (Wang et al. 2012), with only $3.3 \%$ men and $1.6 \%$ women exceeding tolerable weekly intake (TWI) set by the European Food Safety Authority (EFSA) (European Food Safety Authority 2011). In the current study, the cumulative mean intake of cadmium was similar to the findings from other studies in China (He et al. 2013; Yuan et al. 2014). More than $60 \%$ of participants had an estimated cadmium intake above the TWI level.

\section{Dietary Patterns and CKD}

In the Chinese population, several studies have assessed the association between dietary patterns and $\mathrm{HU}$ or serum uric acid. A positive association between meat rich dietary pattern has been reported in several studies in mainland China (He et al. 2017; Liu et al. 2018; Xia et al. 2018; Zhang et al. 2012). The positive association between the traditional dietary pattern and $\mathrm{HU}$ in the current study is consistent with the aforementioned Chinese studies. However, a study in Taiwan reported no significant association between dietary patterns and serum uric acid (Tsai et al. 2012). A uric acidprone pattern is generally characterized by high intake of seafood, meat, beverage and organ meat was positively associated with serum uric acid only before the adjustment of confounding factors.

Several factors may explain the association between the traditional southern dietary pattern and HU. Firstly, the traditional southern dietary pattern has high loadings of pork, fish and poultry. A positive association between the intake of animal food and HU has been found reported in many other studies (Major et al. 2018). In the current study, intake of pork, fish and poultry was also positively associated with HU. Secondly, traditional south pattern was associated with CKD (Shi et al. 2018a). It is well known that impaired kidney function affects the excretion of uric acid and increases the risk of HU. In the current study, the association between the traditional southern dietary pattern and HU was partly mediated by CKD.

Although the traditional southern dietary pattern was positively associated with a high intake of cadmium, the dietary pattern and HU association was not attenuated after adjustment for estimated cadmium intake. It is unlikely that cadmium in the diet can explain the link between traditional dietary pattern and HU.

A null association between the modern dietary pattern and $\mathrm{HU}$ is unexpected. In China, a modern dietary pattern has been shown to be positively associated with obesity (Xu et al. 2015). Nonetheless, several major contributors (i.e. egg, milk, and fruit) in the modern dietary pattern have been to be inversely associated with the risk of HU. For example, egg consumption was inversely association with $\mathrm{HU}$ or serum uric acid levels in Taiwan (Chuang et al. 2011), Croatia (Jeroncic et al. 2010) and the USA (Major et al. 2018). Milk has also been reported to be associated with a lower prevalence of HU or a lower level of serum uric acid (Jeroncic et al. 2010; Major et al. 2018). A higher of consumption of dairy products is associated with a decreased risk of gout (Choi et al. 2004). Furthermore, the modern dietary pattern has been found to be inversely related to CKD in the same study population. It is possible that the adverse effect of this pattern on obesity is counteracted by the inverse relationship with CKD and a lower level of cadmium intake.

No studies on the association between dietary patterns and $\mathrm{HU}$ have incorporated cadmium intake. The inconsistent findings on the association between diet and HU could be partly due to the levels of cadmium in the food in different study populations. As previously reported, $65.4 \%$ of the participants in the CHNS sample had an estimated usual cadmium intake above the EFSA TWI, while $2.9 \%$ were above the WHO provisional tolerable monthly intake (PTMI) (Shi et al. 2018b). The current findings provide additional support for the use of the more conservative EFSA TWI $(2.5 \mu \mathrm{g} /$ $\mathrm{kg} \mathrm{BW} /$ week $)$, rather than the PTMI set by WHO $(25 \mu \mathrm{g} / \mathrm{kg}$ body weight/month) (Shi et al. 2018b).

\section{Strength and Limitations}

The study has several strengths. Firstly, there are multiple measures of dietary intake based on 3-day dietary recall over 18 years. The use of cumulative dietary patterns in the study may reduce misclassification based on a single 3-day dietary recall. Secondly, a variety of potential confounding factors have been adjusted, including health conditions and biomarkers of inflammation. 
One of the main limitations of the study is the crosssectional nature of the current analysis as serum uric acid was only assessed in 2009. Causation cannot be established. However, the findings of the association between dietary pattern and $\mathrm{HU}$ are in line with previous regional studies conducted in China. The food cadmium concentration used to calculate the cadmium intake between 1991 and 2009 is based on national average levels measured in 2012. It may limit the validity of the association between dietary cadmium intake and HU as the food cadmium levels, especially for rice cadmium concentration, vary substantially by regions (He et al. 2013; Yuan et al. 2014) and time. Furthermore, there are no biomedical measurements of cadmium in CHNS to validate the estimated intakes. However, dietary cadmium intake is related to urine cadmium in a study conducted in Shanghai (He et al. 2013). Further studies using blood or urine cadmium measures are needed to examine the role of dietary cadmium in the aetiology of $\mathrm{HU}$ in China.

\section{Novelty and Significance}

As mentioned above, there are a few studies on the association between cadmium (blood or urine) (Sun et al. 2017) or dietary patterns (Liu et al. 2018; Xia et al. 2018; Zhang et al. 2012) and hyperuricemia in China. Most of the studies were regional with one exposure variable (i.e. either cadmium or dietary pattern) and could not answer the following two questions: (1) can the association between cadmium (in the blood or urine) and HU be explained by dietary pattern? (2) Is the association between dietary pattern and HU partly due to cadmium intake? The current study recruited participants from nine provinces in China, and importantly, two related exposure variables (cadmium intake and dietary patterns) were examined for their associations with HU. It gives a whole picture of the interplay of cadmium intake and dietary patterns with HU.

\section{Conclusion}

In conclusion, traditional dietary pattern and cadmium intake are associated with $\mathrm{HU}$ in the Chinese population independent of known HU risk factors. The association between dietary patterns cannot be explained by cadmium contamination of the food supply. Cadmium intake is positively associated with $\mathrm{HU}$, which is mainly due to traditional dietary pattern.

Acknowledgements This research uses data from China Health and Nutrition Survey (CHNS). I thank the National Institute for Nutrition and Health, China Center for Disease Control and Prevention, Carolina Population Center (P2C HD050924, T32 HD007168), the University of North Carolina at Chapel Hill, the NIH (R01-HD30880, DK056350,
R24 HD050924, and R01-HD38700) and the NIH Fogarty International Center (D43 TW009077, D43 TW007709) for financial support for the CHNS data collection and analysis files from 1989 to 2015 and future surveys, and the China-Japan Friendship Hospital, Ministry of Health for support for CHNS 2009, Chinese National Human Genome Center at Shanghai since 2009, and Beijing Municipal Center for Disease Prevention and Control since 2011.

Author Contributions ZS contributed to the conception, analysis, and interpretation of data; drafting of the report; and have given approval of the final version for publication.

Funding Open Access funding provided by the Qatar National Library.

\section{Compliance with Ethical Standards}

Conflict of interest The authors have no relevant financial interest in this article.

Open Access This article is licensed under a Creative Commons Attribution 4.0 International License, which permits use, sharing, adaptation, distribution and reproduction in any medium or format, as long as you give appropriate credit to the original author(s) and the source, provide a link to the Creative Commons licence, and indicate if changes were made. The images or other third party material in this article are included in the article's Creative Commons licence, unless indicated otherwise in a credit line to the material. If material is not included in the article's Creative Commons licence and your intended use is not permitted by statutory regulation or exceeds the permitted use, you will need to obtain permission directly from the copyright holder. To view a copy of this licence, visit http://creativecommons.org/licenses/by/4.0/.

\section{References}

Arrebola JP et al (2019) Associations of multiple exposures to persistent toxic substances with the risk of hyperuricemia and subclinical uric acid levels in BIOAMBIENT.ES study. Environ Int 123:512-521. https://doi.org/10.1016/j.envint.2018.12.030

Choi HK, Curhan G (2004) Beer, liquor, and wine consumption and serum uric acid level: the Third National Health and Nutrition Examination Survey. Arthr Rheumatol 51:1023-1029. https://doi. org/10.1002/art.20821

Choi HK, Atkinson K, Karlson EW, Willett W, Curhan G (2004) Purine-rich foods, dairy and protein intake, and the risk of gout in men. N Engl J Med 350:1093-1103. https://doi.org/10.1056/ NEJMoa035700

Choi JW, Ford ES, Gao X, Choi HK (2008) Sugar-sweetened soft drinks, diet soft drinks, and serum uric acid level: the Third National Health and Nutrition Examination Survey. Arthr Rheumatol 59:109-116. https://doi.org/10.1002/art.23245

Chuang SY, Lee SC, Hsieh YT, Pan WH (2011) Trends in hyperuricemia and gout prevalence: nutrition and Health Survey in Taiwan from 1993-1996 to 2005-2008. Asia Pac J Clin Nutr 20:301-308

European Food Safety Authority (2011) Statament on tolerable weekly intake for cadmium. EFSA J 9:1975. https://doi.org/10.2903/j. efsa.2011.1975

Ferraro PM, Costanzi S, Naticchia A, Sturniolo A, Gambaro G (2010) Low level exposure to cadmium increases the risk of chronic kidney disease: analysis of the NHANES 1999-2006. BMC Public Health 10:304. https://doi.org/10.1186/1471-2458-10-304 
He P et al (2013) Exposure assessment of dietary cadmium: findings from Shanghainese over 40 years China. BMC Public Health 13:590. https://doi.org/10.1186/1471-2458-13-590

He F, Wang LL, Yu XL (2017) Dietary patterns associated hyperuricemia among Chinese aged 45 to 59 years: an observational study. Medicine (Baltimore) 96:e9248. https://doi.org/10.1097/ MD.0000000000009248

Inker LA et al (2012) Estimating glomerular filtration rate from serum creatinine and cystatin C. N Engl J Med 367:20-29. https://doi. org/10.1056/NEJMoa1114248

Jarup L et al (2000) Low level exposure to cadmium and early kidney damage: the OSCAR study. Occup Environ Med 57:668-672

Jeroncic I, Mulic R, Klismanic Z, Rudan D, Boban M, Zgaga L (2010) Interactions between genetic variants in glucose transporter type 9 (SLC2A9) and dietary habits in serum uric acid regulation. Croat Med J 51:40-47

Jung W, Kim Y, Lihm H, Kang J (2019) Associations between blood lead, cadmium, and mercury levels with hyperuricemia in the Korean general population: a retrospective analysis of populationbased nationally representative data. Int J Rheum Dis. https://doi. org/10.1111/1756-185X.13632

Juraschek SP, Gelber AC, Choi HK, Appel LJ, Miller ER 3rd (2016) Effects of the dietary approaches to stop hypertension (DASH) diet and sodium intake on serum uric acid. Arthr Rheumatol 68:3002-3009. https://doi.org/10.1002/art.39813

Kontogianni MD, Chrysohoou C, Panagiotakos DB, Tsetsekou E, Zeimbekis A, Pitsavos C, Stefanadis C (2012) Adherence to the mediterranean diet and serum uric acid: the ATTICA study. Scand J Rheumatol 41:442-449. https://doi.org/10.3109/03009 742.2012 .679964

Krishnan E, Lingala B, Bhalla V (2012) Low-level lead exposure and the prevalence of gout: an observational study. Ann Intern Med 157:233-241. https://doi.org/10.7326/0003-4819-157-4-20120 8210-00003

Li X, He T, Yu K, Lu Q, Alkasir R, Guo G, Xue Y (2018) Markers of iron status are associated with risk of hyperuricemia among chinese adults: nationwide population-based study. Nutrients 10:191. https://doi.org/10.3390/nu10020191

Lin J, Fung TT, Hu FB, Curhan GC (2011) Association of dietary patterns with albuminuria and kidney function decline in older white women: a subgroup analysis from the Nurses' Health Study. Am J Kidney Dis 57:245-254. https://doi.org/10.1053/j. ajkd.2010.09.027

Liu B et al (2011) The prevalence of hyperuricemia in China: a meta-analysis. BMC Public Health 11:832. https://doi. org/10.1186/1471-2458-11-832

Liu X et al (2018) Association of dietary patterns and hyperuricemia: a cross-sectional study of the Yi ethnic group in China. Food Nutr Res. https://doi.org/10.29219/fnr.v62.1380

Major TJ, Topless RK, Dalbeth N, Merriman TR (2018) Evaluation of the diet wide contribution to serum urate levels: meta-analysis of population based cohorts. BMJ 363:k3951. https://doi. org/10.1136/bmj.k3951

Nie X et al (2016) Blood cadmium in Chinese adults and its relationships with diabetes and obesity. Environ Sci Pollut Res Int 23:18714-18723. https://doi.org/10.1007/s11356-016-7078-2

Nuki G, Simkin PA (2006) A concise history of gout and hyperuricemia and their treatment. Arthr Res Ther 8:S1. https://doi. org/10.1186/ar1906
Satarug S, Garrett SH, Sens MA, Sens DA (2010) Cadmium, environmental exposure, and health outcomes. Environ Health Perspect 118:182-190. https://doi.org/10.1289/ehp.0901234

Shi Z, Taylor AW, Riley M, Byles J, Liu J, Noakes M (2018a) Association between dietary patterns, cadmium intake and chronic kidney disease among adults. Clin Nutr 37:276-284. https://doi. org/10.1016/j.clnu.2016.12.025

Shi Z, Taylor AW, Riley M, Byles J, Liu J, Noakes M (2018b) Cadmium intake and chronic kidney disease: response to Kawada $\mathrm{T}$. Clin Nutr 37:1774. https://doi.org/10.1016/j.clnu.2018.06.976

Sun $\mathrm{H}$ et al (2017) Cadmium exposure and its association with serum uric acid and hyperuricemia. Sci Rep 7:550. https://doi. org/10.1038/s41598-017-00661-3

Tsai YT et al (2012) Relationship between dietary patterns and serum uric acid concentrations among ethnic Chinese adults in Taiwan. Asia Pac J Clin Nutr 21:263-270

Wang J, Pan Y, Hong Y, Zhang QY, Wang XN, Kong LD (2012) Quercetin protects against cadmium-induced renal uric acid transport system alteration and lipid metabolism disorder in rats evid based complement. Alternat Med 2012:548430. https://doi. org/10.1155/2012/548430

Wu J et al (2017) Hyperuricemia and clustering of cardiovascular risk factors in the Chinese adult population. Sci Rep 7:5456. https:// doi.org/10.1038/s41598-017-05751-w

Xia Y et al (2018) A dietary pattern rich in animal organ, seafood and processed meat products is associated with newly diagnosed hyperuricaemia in Chinese adults: a propensity score-matched case-control study. Br J Nutr 119:1177-1184. https://doi. org/10.1017/S0007114518000867

Xu X, Hall J, Byles J, Shi Z (2015) Dietary pattern is associated with obesity in older people in China: data from China Health and Nutrition Survey (CHNS). Nutrients 7:8170-8188. https://doi. org/10.3390/nu7095386

Yan S et al (2012) The expanding burden of cardiometabolic risk in China: the China Health and Nutrition Survey. Obes Rev 13:810 821. https://doi.org/10.1111/j.1467-789X.2012.01016.x

Yuan X, Wang J, Shang Y, Sun B (2014) Health risk assessment of cadmium via dietary intake by adults in China. J Sci Food Agric 94:373-380. https://doi.org/10.1002/jsfa.6394

Zhai FY, Du SF, Wang ZH, Zhang JG, Du WW, Popkin BM (2014) Dynamics of the Chinese diet and the role of urbanicity, 19912011. Obes Rev 15(Suppl 1):16-26. https://doi.org/10.1111/ obr. 12124

Zhang M et al (2012) Major dietary patterns and risk of asymptomatic hyperuricemia in Chinese adults. J Nutr Sci Vitaminol (Tokyo) 58:339-345

Zhu Y, Pandya BJ, Choi HK (2011) Prevalence of gout and hyperuricemia in the US general population: the National Health and Nutrition Examination Survey 2007-2008. Arthr Rheumatol 63:3136-3141. https://doi.org/10.1002/art.30520

Publisher's Note Springer Nature remains neutral with regard to jurisdictional claims in published maps and institutional affiliations. 